\title{
Influence du taux d'hémoglobine sur la réponse à la radiothérapie des cancers du col de l'utérus : étude rétrospective de 31 cas au CHU de Dakar
}

\author{
Mamadou SOUMBOUNDOU ${ }^{1}$, Gora MBAYE ${ }^{1 *}$, Ahmadou DEM ${ }^{2}$, Mamadou DIENG $^{2,1}$ \\ Louis Augustin D. DIOUF ${ }^{1}$, Boucar NDONG ${ }^{3}$, Alphonse R. DJIBOUNE, \\ Pape M. SY ${ }^{1}$ et Mounibé DIARRA ${ }^{1}$ \\ ${ }^{1}$ Laboratoire de Physique et de Biophysique Pharmaceutique, Faculté de Médecine, \\ de Pharmacie et d'Odontologie de Dakar, Sénégal. \\ ${ }^{2}$ Service de Cancérologie et de Radiothérapie de l'Hôpital Aristide Le Dantec de Dakar, Sénégal. \\ ${ }^{3}$ Laboratoire de Biophysique Médicale et de Médecine Nucléaire, Faculté de Médecine, \\ de Pharmacie et d'Odontologie de Dakar, Sénégal. \\ *Auteur correspondant ; E-mail :mbaygo@yahoo.fr ; soumboun@hotmail.com ; mounbeni@yahoo.fr; \\ Tel : $775345706,775525686,775022772$
}

\section{RESUME}

Le cancer du col de l'utérus est actuellement le deuxième cancer gynécologique après celui de l'endomètre. Près de 50\% des cas diagnostiqués à travers le monde pourraient profiter de la radiothérapie dont l'efficacité dépend en partie du taux d'hémoglobine. Ainsi, nous avons entrepris d'évaluer l'influence du taux d'hémoglobine sur la réponse à la radiothérapie des cancers du col de l'utérus. Il s'agit d'une étude rétrospective de janvier 2006 à janvier 2009 portant sur 31 dossiers de patientes admises pour un cancer du col de l'utérus et ayant bénéficié d'une radiothérapie exclusive. L'âge moyen des patientes était de 53 ans. Les tranches d'âge les plus représentées étaient celles de 44 à 55 ans et de 55 à 66 ans avec respectivement 16 et 12 patientes. Plus de la moitié des patientes 61,29\% étaient anémiées en début de traitement. Des doses élevées de radiations étaient retrouvées chez les patientes anémiées. En effet, certains auteurs ont montré que les patientes anémiées avant de subir une radiothérapie développaient une radiorésistance tumorale liée à l'hypoxie tissulaire expliquant ces doses élevées. Ces résultats suggèrent une collaboration entre radiothérapeutes et biologistes pour un diagnostic précoce (clinique, biologique) de l'anémie et un suivi biologique des patientes pendant leur traitement afin d'éviter les radiorésistances tumorales et les effets radio-induits tardifs.

(C) 2014 International Formulae Group. All rights reserved.

Mots clés : Anémie, cancer, col utérin, radiothérapie.

\section{INTRODUCTION}

Le cancer du col de l'utérus est actuellement le deuxième cancer gynécologique, après celui de l'endomètre. $\mathrm{Ce}$ cancer est une pathologie rare, devenue moins fréquente dans les pays industrialisés, mais qui demeure la principale tumeur maligne des pays en voie de développement (Marret et al., 2009). Son incidence standardisée dans la population mondiale est égale à 9,5 / 100 000/ an, ce qui constitue 1500 nouveaux cas annuels (Barranger et al., 2005 ; Resbeut et al., 2003). Au Sénégal, il n'y a pas de statistiques disponibles du fait de l'absence 
d'un registre des cancers. Les seules statistiques disponibles concernent les malades qui viennent faire des dépistages avec l'archivage des doubles des résultats.

A l'heure actuelle, on estime que sur l'ensemble des cas de cancer diagnostiqués à travers le monde, $50 \%$ des patients pourraient profiter de la radiothérapie à une étape ou à une autre de l'évolution de leur maladie (Garden et al., 1996). L'efficacité de la radiothérapie dépend du taux d'hémoglobine du patient. En effet, de nombreuses études ont montré que l'anémie, fréquente chez les patients souffrant de cancer, favorise la radiorésistance tumorale par réduction de la production de radicaux libres et de dommages radio-induits de 1 'ADN, provoquant ainsi une meilleure survie cellulaire (Dunst et al., 2008; Dunst et al., 2003a; 2003b ; Hoeckel et al., 2001 ; Rofstad et al., 2000 ; Vaupel et al., 1989). C'est dans ce contexte que nous avons entrepris cette étude afin d'évaluer l'influence du taux d'hémoglobine sur la réponse à la radiothérapie des cancers du col de l'utérus.

\section{MATERIEL ET METHODES}

Il s'agit d'une étude rétrospective de janvier 2006 à janvier 2009 portant sur 51 dossiers de patientes admises pour un cancer du col de l'utérus à l'Institut Curie du CHU Aristide Le Dantec pour radiothérapie. Trente et un (31) dossiers complets et exploitables ont été retenus pour l'étude. Seules les patientes ayant bénéficié d'une radiothérapie exclusive ont été incluses dans l'étude. Les variables suivantes ont été répertoriées : âge, résultats après examen clinique (nature de la réponse : complète, partielle, progressive ou stationnaire), grade selon classification FIGO, la dose en fractionnement classique au volume tumoral et taux d'hémoglobine avant radiothérapie $(\mathrm{Hb})$. L'analyse des données a été faite avec le logiciel Epi info version 7.

\section{RESULTATS \\ Résultats globaux \\ L'âge}

L'âge moyen des patientes était de 53 ans avec des extrêmes allant de 22 ans à 80 ans pour une étendue de 58 ans. La tranche d'âge la plus représentée était celle de 44 à 55 ans (Tableau 1, Figure 1).

\section{Pourcentage de patientes anémiées}

La moyenne des taux d'hémoglobine était de $10,05 \mathrm{~g} / \mathrm{dl}$ avec des extrêmes allant de 4,9 à 13,5 g/dl. Dix neuf (19) personnes sur les 31, soit $61,29 \%$ étaient anémiques avec un taux d'hémoglobine $(\mathrm{Hb})$ inférieur à $11 \mathrm{~g} / \mathrm{dl}$.

\section{Doses totales de radiothérapie reçues}

La moyenne de dose reçue par les patientes était égale à 53,87 Gray (Gy) avec des extrêmes allants de 30 à 66 Gy pour une médiane de $50 \mathrm{~Gy}$.

Les doses les plus fréquemment administrées étaient respectivement 30 Gy $(19,35 \%) 50$ Gy $(32,25 \%)$ et 66 Gy $(48,4 \%)$ (Tableau 2).

\section{Taille des tumeurs}

Le Tableau 3 donne les proportions des différentes tailles des tumeurs. La taille moyenne des tumeurs était égale à $4,45 \mathrm{~cm}$. Plus de 54\% des patientes présentaient une taille tumorale égale à $5 \mathrm{~cm}$.

\section{Stades tumoraux}

Le nombre de patientes par stade tumoral était de 7, 6, 8 et 10 respectivement pour les stades I, II, III et IV.

\section{Pourcentage de réponses tumorales}

Le Tableau 4 donne le pourcentage de la nature de la réponse tumorale après radiothérapie. Il ressort de ce tableau que sur 31 patientes, 5 avaient une réponse complète soit $16,16 \%$.

\section{Résultats analytiques}

Taux d'hémoglobine avant radiothérapie et taille tumorale

Le Tableau 5 donne le taux d'hémoglobine bas en fonction de la taille 
tumorale. Ce tableau montre que sur les 31 patientes traitées pour un cancer du col utérin, $45,2 \%$ étaient anémiées, pour une taille tumorale de $5 \mathrm{~cm}$.

\section{Taux d'Hb en fonction du stade tumoral}

Le Tableau 6 donne l'association entre le taux d'hémoglobine bas et le stade tumoral. Nous constatons que plus le stade tumoral est avancé, plus le pourcentage de sujets présentant un taux d'hémoglobine bas est élevé.

Taux d'hémoglobine et la nature de la réponse tumorale

Le Tableau 7 donne le nombre de réponse tumorale en fonction du taux d'Hb. Nous constatons que chez les anémiques, la réponse n'est complète que chez 04 patientes $(21,05 \%)$.

\section{Taille tumorale et réponse complète}

Le Tableau 8 donne la répartition des réponses complètes après radiothérapie en fonction de la taille tumorale. Nous constatons que la régression tumorale est inversement proportionnelle à la taille tumorale.

Dose totale de radiations reçue et réponse complète

Le Tableau 9 donne la répartition des réponses complètes après radiothérapie en fonction de la dose totale de radiations reçue. Nous constatons que l'obtention d'une réponse complète est assujettie à l'usage d'une dose totale importante de radiations (66 Gy).

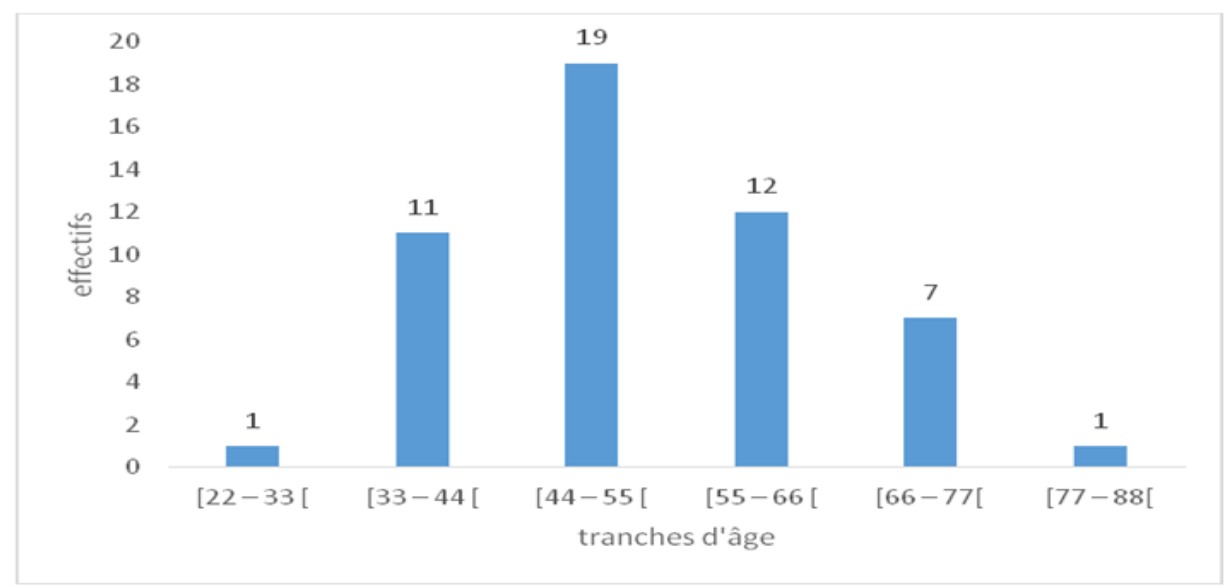

Figure 1 : Nombre de patientes par tranche d'âge.

Tableau 1: Age des patientes.

\begin{tabular}{lcc}
\hline Ages & Effectifs & Pourcentage (\%) \\
\hline$[22-33[$ & 01 & 1,96 \\
{$[33-44[$} & 11 & 21,57 \\
{$[44-55[$} & 19 & 37,25 \\
{$[55-66[$} & 12 & 23,53 \\
{$[66-77[$} & 07 & 13,73 \\
{$[77-88[$} & 01 & 1,96 \\
\hline
\end{tabular}

Tableau 2 : Fréquence des doses. 


\begin{tabular}{lcc}
\hline Doses (Gy) & Effectifs & Pourcentages $(\%)$ \\
\hline 30 & 6 & 19,35 \\
50 & 10 & 32,25 \\
66 & 15 & 48,4 \\
Total & 31 & 100 \\
\hline
\end{tabular}

Tableau 3 : Nombre et pourcentage selon la taille tumorale.

\begin{tabular}{lcc}
\hline Tailles tumeurs $(\mathbf{c m})$ & Effectifs & $\begin{array}{c}\text { Pourcentages } \\
(\%)\end{array}$ \\
\hline 3 & 3 & 9,69 \\
4 & 11 & 35,48 \\
5 & 17 & 54,83 \\
Total & 31 & 100,00 \\
\hline
\end{tabular}

Tableau 4 : Nature des réponses après radiothérapie.

\begin{tabular}{lccccc}
\hline Nature réponse & Complète & Partielle & Progression & Stationnaire & Total \\
\hline Effectifs & $\mathbf{5}$ & 10 & 9 & $\mathbf{7}$ & $\mathbf{3 1}$ \\
Pourcentages $(\%)$ & 16,13 & 32,25 & 29,03 & 22,58 & $\mathbf{1 0 0}$ \\
\hline
\end{tabular}

Tableau 5 : Taux $\mathrm{Hb}$ en fonction de la taille tumorale.

\begin{tabular}{ccccc}
\hline \multirow{2}{*}{ Taux Hb bas } & \multicolumn{3}{c}{ Taille tumorale $(\mathbf{c m})$} & \multirow{2}{*}{ Total } \\
\cline { 2 - 4 } & $\mathbf{3}$ & $\mathbf{4}$ & $\mathbf{5}$ & \\
\hline Non & 3 & 6 & 3 & 12 \\
Oui & 0 & 5 & 14 & 19 \\
Total & 3 & 14 & 17 & 31 \\
\hline
\end{tabular}

Tableau 6 : Taux Hb en fonction du stade tumoral.

\begin{tabular}{cccccc}
\hline \multirow{2}{*}{ Taux Hb bas } & \multicolumn{4}{c}{ Stades tumoraux } & Total \\
\cline { 2 - 5 } & I & II & III & IV & \\
\hline Non & 6 & 3 & 2 & 1 & $\mathbf{1 2}$ \\
Oui & $1(5,26 \%)$ & $3(15,78 \%)$ & $6(31,57 \%)$ & $9(47,36 \%)$ & $\mathbf{1 9}$ \\
Total & 7 & 6 & 8 & 10 & $\mathbf{3 1}$ \\
\hline
\end{tabular}


Tableau 7 : Taux $\mathrm{Hb}$ et nature réponses tumorales.

\begin{tabular}{cccccc}
\hline \multirow{2}{*}{$\begin{array}{c}\text { Taux Hb } \\
\text { bas }\end{array}$} & \multicolumn{4}{c}{ Réponses tumorales } & \multirow{2}{*}{ Total } \\
\cline { 2 - 5 } & Complète & Partielle & Progressive & Stationnaire & \\
\hline Non & 1 & 7 & 3 & 1 & $\mathbf{1 2}$ \\
Oui & $4(21,05 \%)$ & $3(15,70 \%)$ & $6(31,58 \%)$ & $6(31,58 \%)$ & $\mathbf{1 9}$ \\
Total & 5 & 10 & 9 & 7 & $\mathbf{3 1}$ \\
\hline
\end{tabular}

Tableau 8: Réponse complète et taille tumorale.

\begin{tabular}{ccccc}
\hline \multirow{2}{*}{$\begin{array}{c}\text { Réponse } \\
\text { complète }\end{array}$} & & Tailles tumorales $(\mathbf{c m})$ & \multicolumn{2}{c}{ Total } \\
\cline { 2 - 4 } Oui & $\mathbf{3}$ & $\mathbf{4}$ & $\mathbf{5}$ & \\
Non & 0 & 1 & 4 & $\mathbf{5}$ \\
Total & 3 & 10 & 13 & $\mathbf{2 6}$ \\
\hline
\end{tabular}

Tableau 9: Dose totale reçue et réponse complète.

\begin{tabular}{ccccc}
\hline \multirow{2}{*}{$\begin{array}{c}\text { Réponse } \\
\text { complète }\end{array}$} & \multicolumn{2}{c}{ Doses totales reçues de radiations (Gray) } & Total \\
\cline { 2 - 4 } Oui & $\mathbf{3 0}$ & $\mathbf{5 0}$ & $\mathbf{6 6}$ & \\
Non & 1 & 4 & 14 & $\mathbf{1 9}$ \\
Total & 5 & 6 & 1 & $\mathbf{1 2}$ \\
\hline
\end{tabular}

\section{DISCUSSION}

L'âge moyen des patientes était de 53 ans avec une étendue de 58 ans $(80-22)$. Cet âge moyen est comparable à celui d'une étude prospective de 114 cas d'anémie chez des Italiennes atteintes d'un carcinome du col de l'utérus localement avancé avec une moyenne d'âge de 52 ans (extrêmes: 25 et 75 ans) (Ferrandina et al., 2006). Cependant, 05 ans auparavant, dans le même institut, l'âge moyen était de 35 ans sur une population d'étude beaucoup plus importante 616 dossiers. Il y a donc une augmentation de l'âge des patientes au moment du diagnostic (Dem et al., 2008).

Les tranches d'âge les plus représentées étaient celle de 44 à 55 ans avec 16 patientes et celle de 55 à 66 ans avec 12 patientes. Le nombre de sujets âgés était représentatif.

Une étude prospective portant sur 75 patientes a confirmé que l'âge peut être un facteur de survenue des cancers du col de l'utérus (Dem et al., 2008), ce qui conforte nos résultats.

L'anémie est un facteur de pronostic défavorable au cours de la radiothérapie des 
cancers. Elle est souvent liée au stade et à la taille tumorale, mais n'est pas un facteur indépendant (Kim et al., 2007).

Dans notre étude, $61,29 \%$ des patientes, soit 19/31 étaient anémiées en début de traitement, confirmant la fréquence de l'anémie au cours des cancers du col de l'utérus. En effet, les patientes non anémiées en début de traitement et qui le devenaient durant la radiothérapie ont un pronostic aussi défavorable que les patientes présentant initialement une anémie (Marchal et al., 2005 ; Grogan et al., 1999).

Par ailleurs, Il existe une corrélation entre le stade tumoral et la fréquence de l'anémie. Des études ont montré que la fréquence de l'anémie augmente avec le stade tumoral avec $25 \%$ en cas de cancer de stade I, $33 \%$ en cas de cancer de stade II et $40 \%$ en cas de cancer de stade III (Dische, 1991). Ces résultats recoupent les nôtres avec respectivement $5,26 \%$ pour le stade I, $15,78 \%$ pour le stade II, $31,57 \%$ pour le stade III et $47,36 \%$ pour le stade IV.

La corrélation entre taille tumorale et anémie montre une augmentation de la fréquence de l'anémie avec la taille de cette dernière. En effet, le nombre de patientes anémiques étaient de 0,5 et 14 respectivement pour des tailles tumorales de 3,4 et $5 \mathrm{~cm}$. Ces résultats ont été retrouvés par d'autres auteurs mettant en évidence une relation directe entre taille tumorale et anémie (Bush et al., 1978).

Notre étude a montré un taux de réponse tumorale complète après radiothérapie satisfaisant pour les patientes non anémiées avant le début du traitement. Ces résultats sont en concordance avec les données de la littérature. En effet, des études récentes ont montré que les patientes initialement anémiées dont le taux d'hémoglobine avait brutalement chuté en dessous de $10 \mathrm{~g} / \mathrm{dl}$, et pour lesquelles l'anémie a été compensée au-delà de $12,5 \mathrm{~g} / \mathrm{dl}$, avaient un taux de réponse tumorale de $88 \%$, tout à fait comparable à celui des patientes non anémiées (Fyles et al., 2000 ; Baalbergen et al., 2004).

Lors de notre étude, les doses élevées ont été retrouvées chez les patientes anémiées.
En effet, certains auteurs ont montré que les patientes anémiées avant radiothérapie développaient une radiorésistance tumorale liée à l'hypoxie tissulaire expliquant les doses élevées de radiations (Dunst et al., 2003b).

\section{Conclusion}

Les résultats de notre étude ont montré la fréquence de l'anémie pour les tumeurs de grandes tailles et aux stades avancés.

Par conséquent, vu la fréquence de l'anémie chez les patientes, il serait donc nécessaire d'individualiser des stratégies de prévention futures.

$\mathrm{Au}$ terme de notre étude, nous proposons, en collaboration avec les radiothérapeutes et oncologues d'une part, un diagnostic précoce (clinique, biologique) de l'anémie et d'autre part, un suivi biologique des patientes pendant leur traitement, qui pourraient contribuer à éviter les radiorésistances tumorales et les effets radioinduits tardifs.

\section{REFERENCES}

Baalbergen A, Ewing-graham PC, Hop WC, Struijk P, Helmerhorst TJ. 2004. Prognostic factors in adenocarcinoma of the uterine cervix. Gynecol. Oncol., 92: 262-267.

Barranger E, Fay S, Cortez A, Uzan S, Daraï E. 2005. Technique et résultats du prélèvement du ganglion sentinelle dans les cancers du col et du corps de l'utérus. EMC-Gynécologie Obstétrique, 2: 99109.

Bush RS, Jenkin RD, Allt WE, Beale FA, Bean H, Dembo AJ, Pringle JF. 1978. Definitive evidence for hypoxic cells influencing cure in cancer therapy. $\mathrm{Br} J$. Cancer, 37: 302-306.

Dem A, Dieng MM,Traore B, Gaye M, Diop M, Toure P. 2008. Les carcinomes épidermoïdes du col utérin à l'Institut du cancer de Dakar. Cahiers Santé, 18(1): 31-33.

Dische S. 1991. Radiotherapy and anaemia: the clinical experience. Radiother. Oncol., 20: 35-40. 
Dunst J, Kuhnt T, Strauss HG, Krause U, Peltz T, Koelbl H, Hansgen G. 2003. Anemia in cervical cancers: impact on survival, patterns of relapse, and association with hypoxia and angiogenesis. Int. J. Radiat. Oncol. Biol. Phys., 56: $778-787$.

Dunst J, Molls M. 2008. Relationship Between Anemia and Tumor Hypoxia. Recombinant Human Erythropoietin in Clinical Oncology. Nowrousian MR (ed). Springer-Verlag: Vienne; 866.

Dunst J, Stadler P, Becker A, Lautenschläger C, Peltz T, Hansgen G, Molls M, Kuhnt T. 2003. Tumor volume and tumor hypoxia in head and neck cancers. The amount of the hypoxic volume is important. Strahlenther Onkol., 179: 521526.

Ferrandina G, Distefano M, Smaniotto D, Morganti A, Paglia A, Macchia G, Corvari B, Lorusso D, Scambia G. 2006. Anemia in patients with locally advanced cervical carcinoma administered preoperative radiochemotherapy: Association with pathological response to treatment and clinical outcome. Gynecologic Oncology, 103: 500-505.

Fyles A, Milosevic M, Pintilie M, Syed A, HilL R. 2000. Anemia, hypoxia and transfusion in patients with cervix cancer: a review. Radiother Oncol., 57: 13-19.

Garden FH, Gillis TA. 1996. Principles of Cancer Rehabilitation. Saunders: Philadelphia; 1199-1214.

Grogan M, Thomas GM, Melamed I, Wong F L, Pearcey RG, Joseph PK, Portelance L, Crook J, Jones KD. 1999. The importance of hemoglobin levels during radiotherapy for carcinoma of the cervix. Cancer, 86: 1528-1536.
Hoeckel M, Vaupel P. 2001. Tumor hypoxia: definition and current clinical, biological and molecular aspects. J. Natl. Cancer Inst., 93: 266-276.

Kim YT, Kim SW, Yoon BS, Nahm EJ, Kim SH, Kim JH, Kim J W. 2007. Effect of intravenously administered iron sucrose on the prevention of anemia in the cervical cancer patients treated with concurrent chemoradiotherapy. Gynecologic Oncology, 107: 199-204.

Marchal C, Rangeard L, Brunaud C. 2005. Impact de l'anémie sur les traitements des cancers du col utérin. Cancer/Radiothérapie, 9: 87-95.

Marret H, Barillot I, Rolland Y, Leveque J. 2009. Apport de l'échographie Doppler avec injection de contraste pour la prise en charge des cancers du col de l'utérus de stades IB et II par chimioradiothérapie concomitante. Cancer/Radiothérapie, 13: 515-519.

Resbeut M, Fondrinier E, Fervers B, Haiemeder C, Bataillard A, Lhomme C, Asselain B, Basuyau JP. 2003. Standards, options et recommandations. Cancers invasifs du col utérin, stades non métastatiques. Bull cancer, 90: 333-346.

Rofstad EK, Sundfor K, Lyng H. 2000. Hypoxia-induced treatment failure in advanced squamous cell carcinoma of the uterine cervix is primarily due to hypoxia-induced radioresistance rather than hypoxia-induced metastases. $\mathrm{Br} J$ Cancer, 83: 354-359.

Vaupel P, Kallinowski F, Okunieff P. 1989. Blood flow, oxygen and nutriment supply, and metabolic environment of human tumors: a review. Cancer Res, 49: 649-665. 